\title{
Oxidation Stiffening of PDMS Microposts
}

\author{
Joo Yong $\operatorname{Sim}^{a \dagger}$, Rebecca E. Taylor ${ }^{a}$, Tom Larsen ${ }^{a}$, Beth L. Pruitt ${ }^{a \#}$ \\ a Department of Mechanical Engineering, Stanford University, Stanford, CA 94305, USA \\ ${ }^{\dagger}$ Present address: Bio-Medical IT Convergence Research Division, Electronics and \\ Telecommunications Research Institute, Daejeon, 305-700, Republic of Korea \\ ${ }^{\text {\#} C o r r e s p o n d i n g ~ A u t h o r: ~ B e t h ~ L . ~ P r u i t t ~(e m a i l: ~ p r u i t t @ s t a n f o r d . e d u, ~ a d d r e s s: ~} 496$ Lomita Mall \\ Room 213, Durand Building, Stanford, CA 94305-4040)
}

\begin{abstract}
This paper presents guidance for analyzing the stiffness changes of micropost arrays after oxidation. Numerous mechanobiology studies exploit micropost arrays to quantify cell-generated micropost displacements and traction forces. Cell adhesion requires surface functionalization typically facilitated by an oxidation step, which can form a glassy skin. We estimate the material properties of this skin, analytically calculate the stiffness of oxidized microposts and compare it with direct measurements. We find correction factors increase exponentially with decreasing micropost diameter. Ignoring this oxidation stiffening of microposts results in more than $200 \%$ underestimation in micropost stiffness as well as the cellular traction forces extracted from micropost deflections.
\end{abstract}

Keywords: traction force, mechanotransduction, rigidity sensing

\section{Introduction}

Cell contractile forces play an important role in controlling morphogenesis during development [1] and directing the lineage of differentiation [2], modulating cell proliferation [3] and inflammatory response [4]. Mechanotransduction through mechanical cues is implicated in these processes, e.g., the role of substrate stiffness [5,6] or tissue geometry [7,8]. To modulate the physical parameters of the environment and quantify the nanoNewton-level forces generated by cells, two dominant traction force microscopy (TFM) methods are commonly used [2]: flat and deformable substrates with embedded fiducial tracking [9] and elastomeric substrates with arrays of micro-/nano-posts [10]. Flat TFM substrates require several assumptions and solution of a complex inverse problem to map fiducial displacements to surface tractions. Whereas, micropost arrays exploit beam bending of digitized "sensors" and micropost tip deflections are converted directly to forces from the micropost bending stiffness or spring constant. Typically, the micropost spring constant and effective substrate stiffness are modulated by altering micropost height [6]. Comparing between and within these two TFM methods across different labs is challenging because of uncertainties in quantifying traction forces at the nanoNewton scale. To enhance force precision and accuracy using microposts, several researchers have proposed improved estimation of stiffness using sophisticated models of micropost mechanics [11] and material viscoelastic properties [12] or physical calibration using precision force sensors such as pulled glass micro-pipettes [10], atomic force microscopy (AFM) $[\mathbf{1 3 , 1 4 ]}$ and MEMS (micro-electricalmechanical-systems) force sensors $[\mathbf{1 1}, \mathbf{1 5}, \mathbf{1 6}]$.

TFM micropost arrays are typically fabricated in polydimethylsiloxane (PDMS). Due to the hydrophobic nature of PDMS, surface modification is required to make PDMS microposts hydrophilic and amenable to protein functionalization for cell adhesion [17]. Surface modification typically includes oxidation of the PDMS surface to introduce functional hydroxyl groups that temporarily render the 
surface hydrophilic [18]. Previous studies have used X-ray photoelectron spectrometry (XPS) [19], scanning electron microscopy (SEM) [20] or transmission electron microscopy (TEM) [21], atomic force microscopy (AFM) [22], and film buckling [23-26] to show that the oxidation of bulk PDMS forms a thin silicate layer on the surface. However, micropost stiffness characterization $[\mathbf{1 0 , 1 1 , 1 3 - 1 6 , 2 7 , 2 7 ]}$ is typically conducted only before oxidation and neglects oxidation effects. The Young's modulus of PDMS ranges from 1 to 2 $\mathrm{MPa}$ but the modulus of glass is more than $1 \mathrm{GPa}$. This difference motivates our hypothesis that oxidation has an appreciable impact and our investigation of the effect on the mechanics of oxidized PDMS microposts. This study provides: 1) characterization of PDMS micropost stiffness before and after oxidation using a calibrated force sensor, 2) assessments of the mechanical properties of the oxidized PDMS layer, and 3) a comparison between the theoretical model of the oxidized PDMS microposts as a composite beam and the measurement of the stiffness change after oxidation.

\section{Results}

\section{Characterization of Micropost Array Stiffening due to Plasma Oxidation.}

To evaluate stiffening of microposts due to plasma oxidation, we measured the stiffness of microposts before and after oxygen plasma treatment using a piezoresistive force sensor. We fabricated micropost arrays by replica molding from an SU-8 mold as described previously [10,15]. In brief, 10:1 PDMS was poured on an SU-8 master with holes having a 5 $\mu \mathrm{m}$ diameter, $10 \mu \mathrm{m}$ deep and $10 \mu \mathrm{m}$ pitch, degassed in a vacuum chamber to remove bubbles for $30 \mathrm{~min}$ and cured at $80{ }^{\circ} \mathrm{C}$ for $12 \mathrm{~h}$. Then we peeled off the PDMS micropost array and treated with oxygen plasma $\left(\mathrm{O}_{2}\right.$ Barrel Asher, Branson) at $80 \mathrm{~W}$ and 0.45 Torr. The diameter and length of the cylindrical plasma chamber were $21 \mathrm{~cm}$ and $35 \mathrm{~cm}$, respectively (total volume of $1.212 \times 10^{-2} \mathrm{~m}^{3}$ ).

We observed that the micropost stiffness increased by a factor of 2-10 after oxygen plasma treatment depending on the time of treatment (Figure 1). To confirm that this was not due to dimensional changes from etching of PDMS, we measured the height and diameter of microposts as a function of oxidation time using a scanning electron microscope. Microposts did not exhibit measurable shortening in heights or increase in diameters.

To estimate the thickness and elastic modulus of oxidized surface layers we used buckling instability of a stiff thin film on a relatively soft thick substrate under compressive strain [20]. To induce film wrinkling on the PDMS, we prepared 1 $\mathrm{mm}$ thick, $10 \mathrm{~mm}$ wide, $60 \mathrm{~mm}$ long PDMS samples and stretched them from $4 \%$ to $12 \%$. We then treated the stretched
PDMS samples with oxygen plasma over a range of $15 \mathrm{~s}$ to 10 $\min$. The strain was then released manually at a rate of $0.01 / \mathrm{s}$.

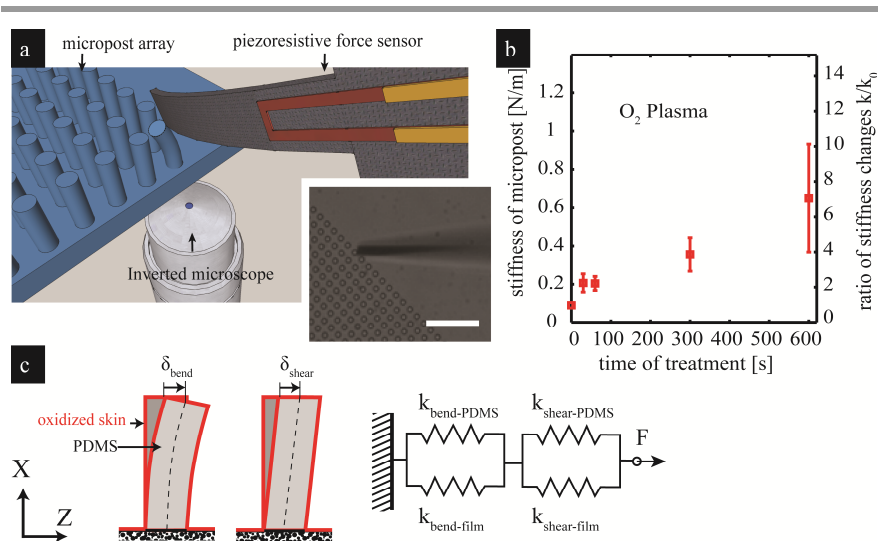

Figure 1 Micropost stiffness increases after the oxidation treatment of the oxygen plasma. (a) The measurement set-up of micropost stiffness includes the piezoresistive MEMS force sensor and inverted microscope with 40x objective. The insert shows the microscope view during the measurement (scale bar, $40 \mu \mathrm{m}$ ) (b) Stiffness of micropost as a function of oxygen plasma treatment time, error bars are S.E.M. $(n=10$ for each measurement point) (c) Diagram of the micropost deformation model. The contributions of bending and shear are modeled as series springs, and the contributions of the stiff skin and PDMS in each mode of deformation are modeled as parallel springs.

Using buckling instability of a stiff thin film on a relatively soft thick substrate under compressive strain [20], we estimated the thickness and elastic modulus of oxidized surface layers. The compressive strain induced periodic wrinkles in the oxidized skin by releasing pre-strain after oxygen plasma treatment (Figure 2a). Assuming the compressive strain equals the strain released from stretching, the amplitude and wavelength of the wrinkles can be modeled by the compressive strain, the film thickness, and elastic modulus of the film. We used models describing the wavelength and amplitude of wrinkling from N. Bowden, et al. [20] and Lacour et al. [28]

$$
\lambda_{0}=\frac{\pi h_{f}}{\sqrt{\varepsilon_{\text {critical }}}}
$$

and

$$
A_{0}=h_{f} \sqrt{\frac{\varepsilon}{\varepsilon_{\text {critical }}}-1}
$$

where $\lambda_{0}$ is the wavelength, $A_{0}$ is amplitude, $h_{f}$ is the film thickness and $\varepsilon_{\text {critical }}$ is the critical buckling strain. The critical buckling strain is independent of the film thickness and prestrain and can be expressed as $[\mathbf{2 0 , 2 4 ]}$ 


$$
\varepsilon_{\text {critical }}=\frac{1}{4}\left(3 \frac{E_{s}}{E_{f}}\right)^{\frac{2}{3}}
$$

where $E_{s}$ is the elastic modulus of substrate PDMS and $E_{f}$ is the elastic modulus of film. For computational purposes, we fitted the square of wrinkle amplitude to the pre-strain based on (13). The growth rate of the skin layer was estimated from the relationship between the wavelength of wrinkling versus the oxidation treatment time using (12).

The amplitude and wavelength of the induced wrinkles increased with oxygen plasma treatment time (Figure $2 b$ ). We obtained the critical buckling strain, $\varepsilon_{\text {critical }}=0.042$ based on the squared wrinkle amplitude of samples oxidized with varying strain (Figure 2c). Using this critical strain, we calculated the ratio of the substrate and oxidized film elastic moduli, $E_{f} / E_{s}=43$ and estimated a silicate film growth rate of $20 \mathrm{~nm} / \mathrm{min}$ (Figure 2d).
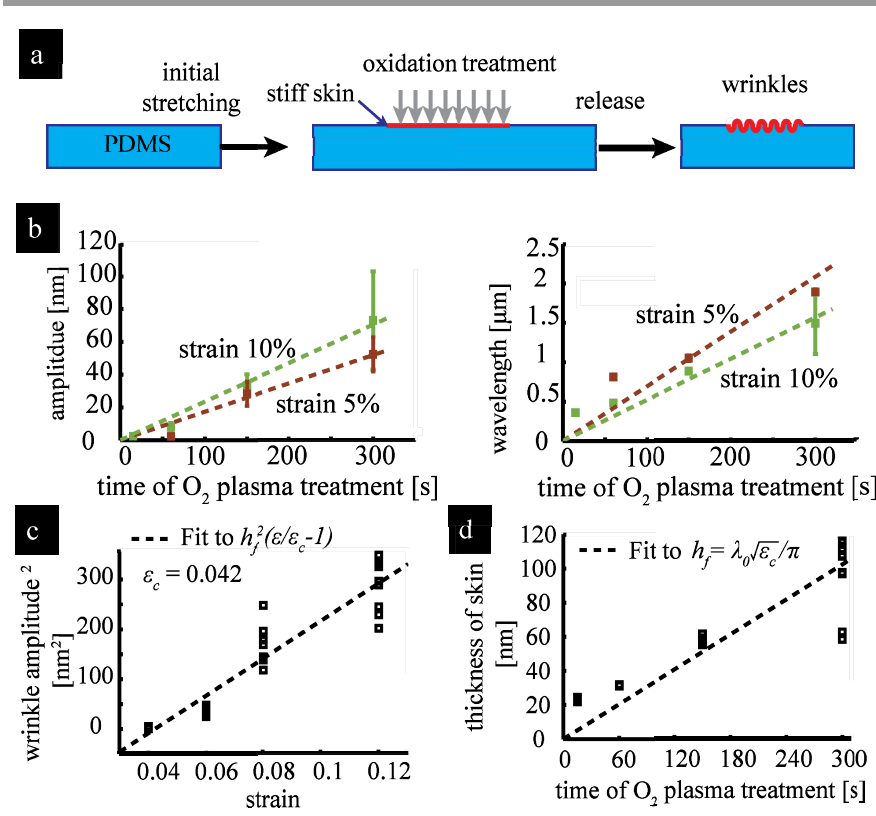

time of $\mathrm{O}_{2}$ plasma treatment $[\mathrm{s}]$

Figure 2 The oxidized surface layer on PDMS induces a highly ordered pattern of wrinkles when the pre-strain is released. (a) PDMS samples are pre-stretched before oxidation and then released to induce film wrinkling. (b) The wrinkling amplitude and wavelength as a function oxygen plasma time. We estimate the amplitude and wavelength of the wrinkles using a Fast Fourier Transform (Matlab function 'fft'). The data are fitted using a linear fit. (c) The square of the wrinkle amplitude depending on strain (from 0.04 to 0.12 ) after $1 \mathrm{~min}$ of oxygen plasma. The square of the amplitude is assumed to be linearly related to the pre-strain applied before oxygen plasma treatment. Least square fit equation (13) yielded a skin thickness of $\left(h_{f}=12.59 \mathrm{~nm}\right)$ and critical strain of $\left(\varepsilon_{c}=0.042\right)$. Given the critical strain, we estimate the Young's modulus ratio between the skin and substrate $\left(E_{f} / E_{s}=43.29\right)$. (d) Skin thickness as a function of plasma treatment time. Assuming the thickness of the skin increases with the time of oxidation treatment, the oxidation thickening rate is $20 \mathrm{~nm} / \mathrm{min}$.
Theoretical Modeling of Micropost with Stiff Skin.

We considered the bending and shear deformation of a beam using Timoshenko's beam theory since the height-to-diameter aspect ratio is smaller than 5:1. For simplicity, we did not take the tilting of the base into account because the stiff skin on the base has a negligible impact on base warping; the skin has small finite thickness and large surface area compared with the base warping effect with a 3D half infinite half-space assumption. Moreover, we estimated that the skin increases the micropost stiffening via base tilting by only $3 \%$ compared to a $200 \%$ increase in overall bending stiffness with the skin using 3D finite element simulations (COMSOL Multiphysics $\left.{ }^{\circledR}\right)$. For transverse beam bending (Figure 1d), we can write the bending moment $M$ and the shear force $Q$ as [29]

$$
\begin{aligned}
& M=\frac{d \theta}{d x} \iint_{A} E z^{2} d A \\
& Q=\left(\frac{d w}{d x}-\theta\right) \iint_{A} G d A
\end{aligned}
$$

where $E$ is the elastic modulus, $G$ is the shear modulus, $w$ is the displacement in the direction of tip loading, $A$ is the area of beam cross section and $\theta$ is the rotation of shearing cross section with beam bending and shear. Assuming isotropic and linear elastic materials, we can write the equation (1) and (2) for our composite of core and skin by

$$
\begin{aligned}
& M=\frac{d \theta}{d x}\left(E_{1} I_{1}+E_{2} I_{2}\right) \\
& Q=\left(\frac{d w}{d x}-\theta\right)\left(G_{1} A_{1}+G_{2} A_{2}\right)
\end{aligned}
$$

where the $E_{l}$ is the elastic modulus of the core, $I_{l}$ is the moment of inertia of the circular core, $E_{2}$ is the elastic modulus of the skin, $I_{2}$ is the moment of inertia of the skin as a hollow cross-section, $G_{l}$ is the shear modulus of the core, $A_{I}$ is the cross section area of the core, $G_{2}$ is the shear modulus of the skin and $A_{2}$ is the cross section of the skin. Equation (2) assumes that shear stress $\tau_{x z}$ stays constant across the area of the cross-section. However, the exact distribution of shear stress $\tau_{x z}$ is not constant so we need to compensate the difference by multiplying with the shear correction factor $K$. We assume that the Timoshenko's shear correction factor of composite is close to that for the case of a circular or of a hollow cylindrical cross-section beam. Then, we can replace equation (4) with

$$
Q=\left(\frac{d w}{d x}-\theta\right)\left(K_{1} G_{1} A_{1}+K_{2} G_{2} A_{2}\right)
$$

where $K_{1}$ is $6(1+v) /(7+6 v)$ and $K_{2}$ is $2(1+v) /(4+3 v)$ and $v$ is Poisson ratio [29]. 
To calculate the displacement by the tip loading of the beam, $F$, the moment $M$ can be expressed by

$$
\begin{aligned}
& M=F(L-x)=\frac{d \theta}{d x}\left(\sum E I\right) \\
& \theta=\frac{-F}{\sum E I}\left(\frac{x^{2}}{2}-L x+C_{1}\right)
\end{aligned}
$$

where $C_{l}=0$ with the boundary condition of $\theta=0$ at $x=0$, since the rotation of the shearing cross section must be zero at the base of the micropost. Inserting (7) into (5) gives us

$$
\begin{aligned}
& Q=\left(\frac{d w}{d x}+\frac{F}{\sum E I}\left(\frac{x^{2}}{2}-L x\right)\right) \sum K G A \\
& w=\frac{Q}{\sum K G A} x-\frac{F}{\sum E I}\left(\frac{x^{3}}{6}-\frac{L x^{2}}{2}+\mathrm{C}_{2}\right)
\end{aligned}
$$

where $C_{2}=0$ with the boundary condition of $w(0)=0$ and $Q=$ $F$, since the post displacement is zero at the base of the micropost and the shear force applied is equal to the loading force on the beam. Thus, the tip displacement of the composite beam, $w(L)$ or $\delta$ is

$$
\delta=\frac{F}{\frac{\sum K G A}{L}}+\frac{F}{3 \sum E I}
$$

and the stiffness of the beam to the tip loading, $k$, has a relationship of

$$
\frac{1}{k}=\frac{\delta}{F}=\frac{1}{\frac{K G_{1} A_{1}}{L}+\frac{K G_{2} A_{2}}{L}}+\frac{1}{\frac{3 E_{1} I_{1}}{L^{3}}+\frac{3 E_{2} I_{2}}{L^{3}}}
$$

where we can consider the two deformation modes of shear and pure bending as a series of springs where two materials are modeled as parallel springs (Figure 1c). We validate our analytical model using COMSOL Multiphysics ${ }^{\circledR}$ for finite element modeling. We assume a nearly incompressible linear elastic material with a Poisson ratio of $(v \approx 0.49)$ as for the theoretical modeling with Timoshenko's beam theory. The mechanical characterization of the oxidized film will be explained in the following section.

\section{Discussion}

To demonstrate the impact of micropost stiffening on cell traction force measurements, we revisited our previously reported data for micropost arrays with stiffness calibrations before oxidation and estimated the oxidation stiffening as a function of treatment time of oxygen plasma (Figure 3a) [15]. Figure $3 \mathrm{~b}$ shows the corrected and uncorrected traction force vectors under a rat neonatal cardiomyocyte at maximal cell contraction for micropost arrays with height, diameter and pitch of $10 \mu \mathrm{m}, 5 \mu \mathrm{m}$ and $5 \mu \mathrm{m}$ respectively. Using oxidation stiffening factors for $30 \mathrm{~s}$ of oxygen plasma from Figure 3a, we revised estimates of the average force per post upwards by a factor of 2.3. Figure $3 \mathrm{c}$ shows the average force per micropost with and without the oxidation-stiffening effect. The average force per post was corrected from $39 \pm 5 \mathrm{nN}$ to $89.7 \pm 11.5 \mathrm{nN}$.

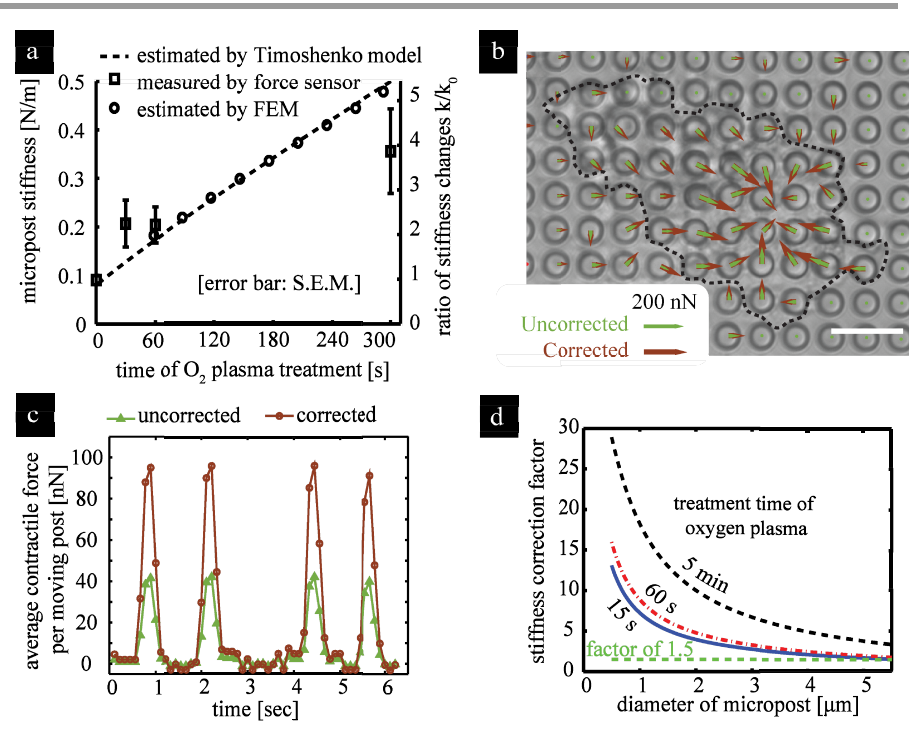

Figure 3 The micropost stiffness changes can be approximated using the mechanical properties of the skin. (a) The measured stiffness of microposts using a MEMS force sensor and the theoretical micropost stiffness based on the Timoshenko model and finite element model for an oxidized micropost. (b) A force measurement of neonatal cardiomyocytes cultured on micropost arrays (scale bar, $10 \mu \mathrm{m}$ ). (c) The average contraction force of the cardiomyocytes before and after considering oxidation stiffening. (d) Correction factors for oxidation stiffening on micropost arrays as a function of micopost diameter.

Our results suggest that the micropost stiffness can change by more than $200 \%$ after oxygen plasma treatment. Thus, oxidation stiffening of microposts must be considered for precise force estimation. These results provide guidance for the design of micropost arrays to measure cell traction forces and for targeting effective substrate rigidities. These correction factors are particularly important when comparing measured forces between different force measurement techniques, e.g., TFM using continuous deformable substrates vs. micropost arrays. With oxidation stiffening, correction factors can become dramatically large. As the diameter of micropost decreases for a given thickness of oxidation layer, the bending stiffness scales with 4th power dependency on micropost radius (Figure 3d). The stiffness of micropost arrays with $1 \mu \mathrm{m}$ diameter can for example increase by over $500 \%$ when the skin thickness is $5 \mathrm{~nm}$ formed by a 15 seconds plasma treatment. 
Our estimate of $E_{f} / E_{s} \approx 43$ for our oxidized surface films is similar to that of $E_{f} / E_{s} \approx 60$ reported by Moon et al. [26]. However, our growth rate estimate of $20 \mathrm{~nm} / \mathrm{min}$ is much larger than their observed rate of $5.64 \mathrm{~nm} / \mathrm{min}$. We hypothesize the large difference in growth rates may be due to the different plasma gas and power conditions (air gas and 18 $\mathrm{W}$ for Moon et al. vs. pure oxygen and $80 \mathrm{~W}$ in this work) [26]. Importantly, we assumed a single, homogeneous layer of oxidized skin but the skin might have a continuous gradient of composite materials from skin to PDMS. We also assumed that film growth rate remains constant during the course of treatment. However, as with oxidation of silicon [30], the growth rate may transition from reaction to diffusion limited as the film becomes a diffusion barrier. The silicate film growth rate is most likely dependent on the power, pressure of oxygen plasma, PDMS composition, curing time and variation among systems.

We suggest following three approaches to apply our findings easily across labs. First, when the oxygen plasma conditions are similar ( $80 \mathrm{~W}, 0.5$ Torr, 30 seconds), one can use our measurement results for the thickness and modulus of silicate film and our model to estimate the correction factor for the desired micropost size. Second, when the oxygen plasma conditions differ significantly, the buckling tests may be repeated to estimate the thickness and modulus of the silicafilm and then apply the model presented here to obtain a correction factor for micropost stiffness. Third, an atomic force microscope, a piezoresistive cantilever force sensor, or similar microsystems can be used for direct calibration of microposts before and after oxidation. The first two approaches have the advantage of application to any micropost geometry (radii and height) using the estimated film parameters and general model (Figure 3d).

We estimated a silicate growth rate of $20 \mathrm{~nm} / \mathrm{min}$ by using the buckling test. A more direct way of estimating thickness would be to use transmission election microscopy to measure the thickness as a function of oxidation time. Béfahy et al. used this approach to investigate the silicate growth for different plasma conditions [21]. Fitting and extrapolating their results to our conditions, the predicted growth rate is $\sim 15 \mathrm{~nm} / \mathrm{min}$ which is in agreement with our result [21].

Speculating that the oxidized surface layer is more brittle than the intact PDMS, we considered the failure mode of the oxide skin. We estimated the maximum stress on the oxidized layer of the micropost using the Timoshenko beam equation. The axial stress $\boldsymbol{\sigma}_{x}$ is given by $\boldsymbol{\sigma}_{x}=-E z d \theta / d x$. From equation (3) and (6), we can expect that the maximum axial stress occurs at the root of the micropost and the top surface of the skin when $z=R$ and $x=L$ where $R$ and $L$ are the radius and length of the micropost. Thus, the maximum axial stress is expressed by $\boldsymbol{\sigma}_{\max }=F L E_{2} R /\left(E_{1} I_{1}+E_{2} I_{2}\right)$. Since we expected the oxidized surface layer to have a higher ultimate tensile strength than PDMS, we estimated a lower bound of the failure mode when the maximum axial stress exceeds the yield strength of PDMS (7.5 MPa from Mata et al. [31]). The equation of maximum axial stress provides the lower bound of the maximum force and maximum displacement before mechanical failure. We obtained the maximum force and displacement per micropost, $720 \mathrm{nN}$ and $6 \mu \mathrm{m}$ respectively, which are 2-5 times larger than what we typically expect for cell-generated force per post $[6,10]$. Fayolle et al. describe the ductile-brittle transition which occurs in polymer oxidation aging due to the increase in chain scission [32]. The thermal and photo-degradation of polymers have also been reported in the context of mechanical failure [32,33]. The critical molar mass, which decreases due to the degradation, is an important parameter to determine the embrittlement [32,33]. However, the embrittlement of plasma-induced oxidized PDMS is beyond the scope of the present work and requires further study.

UV ozone is another common tool used to oxidize and functionalize PDMS surfaces for cell adhesion [6,17]. Since the effect of UV ozone is mild, UV ozone is frequently used as well and typically requires longer treatment than oxygen plasma (5 to $15 \mathrm{~min}$ ). Future investigation of PDMS surface oxidation by UV ozone may be warranted. Our preliminary studies show that a 7 min UV ozone treatment results in a smaller stiffening effects $(50 \%$ increase in stiffness $)$ compared to a $30 \mathrm{~s}$ oxygen plasma treatment (200\% increase). Based on the previous work on the surface characterization of two functionalized surfaces of UV ozone and oxygen plasma [34], the UV ozone may be a desirable alternative to oxygen plasma as it potentially causes less stiffening. A possible method to avoid the stiffening effect would be to use a sacrificial layer such as Poly(N-isopropylacrylamide) [16] to physically protect the PDMS in between microposts by blocking oxygen plasma. This technique has the potential to selectively functionalize the top of the microposts, so the mechanical properties of the microposts would not be affected. To minimize correction factors, it would be ideal to use no oxidation. However, if oxidation is necessary, minimizing treatment time will reduce the correction factor as much as possible. Finally, the oxidized PDMS skin may crack under the strain similar to stretched silicon dioxide on PDMS [35]. The effect of cracks on the oxidized skin in their interaction with the PDMS micropost remains to be further investigated.

\section{Conclusions}

We estimated the elastic modulus and thickness of oxidized layers on PDMS formed by oxygen plasma using the buckling stability of skin. The effect of micropost stiffening due to skin 
formation was described analytically. We also directly calibrated the micropost stiffness after oxidation and compared it with our analytical predictions of oxidation stiffening. The stiffening factor is significant when comparing the absolute values from the literature. Therefore, the correction of micropost stiffness is important for designing micropost arrays and determining the force resolution of the system. The precise modeling and calibration of elastomeric micropost arrays presented here will allow us to improve the applications of micropost arrays and other PDMS-based cantilever sensors [36,37]. Applications for these devices include studies of mechanobiology in stem cell differentiation [6], force cytometry for cardiomyocyte contraction [38], and mapping forces during transmigration of immune cells [39]. This work can also help address the challenges of absolute measurements of cellular level nanoNewton [40]. Further, our work urges the broad interest in the field of flexible PDMS device with an oxidized layer such as stretchable electronics.

\section{Acknowledgements}

This work was funded in part by NSF EFRI MIKS-1136790, NIH R01 HL089027, NIH R01 EB006745, Stanford Bio-X, a Stanford BioX Graduate Fellowship (JS) and the ILJU foundation fellowship (JS). We thank Prof. Charles Steele for fruitful discussions on the mechanics and modeling of composite beams.

[1] M.A. Wozniak, C.S. Chen, Mechanotransduction in development: a growing role for contractility, Nat. Rev. Mol. Cell Biol. 10 (2009) 34-43. doi:10.1038/nrm2592.

[2] I. Schoen, B.L. Pruitt, V. Vogel, The Yin-Yang of Rigidity Sensing: How Forces and Mechanical Properties Regulate the Cellular Response to Materials, Annu. Rev. Mater. Res. 43 (2013) 589-618. doi:10.1146/annurev-matsci-062910-100407.

[3] G. Halder, S. Dupont, S. Piccolo, Transduction of mechanical and cytoskeletal cues by YAP and TAZ, Nat. Rev. Mol. Cell Biol. 13 (2012) 591-600. doi:10.1038/nrm3416.

[4] D. Huh, B.D. Matthews, A. Mammoto, M. MontoyaZavala, H.Y. Hsin, D.E. Ingber, Reconstituting organlevel lung functions on a chip, Science. 328 (2010) 1662-1668. doi:10.1126/science.1188302.

[5] A.J. Engler, S. Sen, H.L. Sweeney, D.E. Discher, Matrix Elasticity Directs Stem Cell Lineage Specification, Cell. 126 (2006) 677-689. doi:10.1016/j.cell.2006.06.044.

[6] J. Fu, Y.-K. Wang, M.T. Yang, R.A. Desai, X. Yu, Z. Liu, et al., Mechanical regulation of cell function with geometrically modulated elastomeric substrates, Nat. Methods. 7 (2010) 733-736. doi:10.1038/nmeth.1487.

[7] M. Théry, Micropatterning as a tool to decipher cell morphogenesis and functions, J. Cell Sci. 123 (2010) 4201-4213. doi:10.1242/jcs.075150.

[8] E.W. Gomez, Q.K. Chen, N. Gjorevski, C.M. Nelson, Tissue geometry patterns epithelial-mesenchymal transition via intercellular mechanotransduction, J. Cell. Biochem. 110 (2010) 44-51.

doi:10.1002/jcb.22545.

[9] C.-M. Lo, H.-B. Wang, M. Dembo, Y. Wang, Cell Movement Is Guided by the Rigidity of the Substrate, Biophys. J. 79 (2000) 144-152. doi:10.1016/S00063495(00)76279-5.

[10] J.L. Tan, J. Tien, D.M. Pirone, D.S. Gray, K. Bhadriraju, C.S. Chen, Cells lying on a bed of microneedles: An approach to isolate mechanical force, Proc. Natl. Acad. Sci. 100 (2003) 1484-1489. doi:10.1073/pnas.0235407100.

[11] I. Schoen, W. Hu, E. Klotzsch, V. Vogel, Probing Cellular Traction Forces by Micropillar Arrays: Contribution of Substrate Warping to Pillar Deflection, Nano Lett. 10 (2010) 1823-1830. doi:10.1021/nl100533c.

[12] I.-K. Lin, Y.-M. Liao, Y. Liu, K.-S. Ou, K.-S. Chen, X. Zhang, Viscoelastic mechanical behavior of soft microcantilever-based force sensors, Appl. Phys. Lett. 93 (2008) 251907. doi:doi:10.1063/1.3056114.

[13] Q. Cheng, M. Almasri, Z. Sun, G.A. Meininger, Micropost array for force mapping of vascular smooth muscle cells, in: 2010 IEEE Sens., 2010: pp. 15831586. doi:10.1109/ICSENS.2010.5690288.

[14] N. Tymchenko, J. Wallentin, S. Petronis, L.M. Bjursten, B. Kasemo, J. Gold, A Novel Cell Force Sensor for Quantification of Traction during Cell Spreading and Contact Guidance, Biophys. J. 93 (2007) 335-345. doi:10.1529/biophysj.106.093302.

[15] K. Kim, R. Taylor, J.Y. Sim, S.-J. Park, J. Norman, G. Fajardo, et al., Calibrated micropost arrays for biomechanical characterisation of cardiomyocytes, IET Micro Nano Lett. 6 (2011) 317-322.

doi:10.1049/mnl.2011.0031.

[16] R.E. Taylor, K. Kim, N. Sun, S.-J. Park, J.Y. Sim, G. Fajardo, et al., Sacrificial layer technique for axial force post assay of immature cardiomyocytes, Biomed. Microdevices. 15 (2013) 171-181. doi:10.1007/s10544012-9710-3.

[17] M.T. Yang, J. Fu, Y.-K. Wang, R.A. Desai, C.S. Chen, Assaying stem cell mechanobiology on microfabricated elastomeric substrates with geometrically modulated rigidity, Nat. Protoc. 6 (2011) 187-213. doi:10.1038/nprot.2010.189.

[18] P.-J. Wipff, H. Majd, C. Acharya, L. Buscemi, J.-J. Meister, B. Hinz, The covalent attachment of adhesion molecules to silicone membranes for cell stretching applications, Biomaterials. 30 (2009) 1781-1789. doi:10.1016/j.biomaterials.2008.12.022.

[19] D. Eon, L. de Poucques, M.C. Peignon, C. Cardinaud, G. Turban, A. Tserepi, et al., Surface modification of Si-containing polymers during etching for bilayer lithography, Microelectron. Eng. 61-62 (2002) 901906. doi:10.1016/S0167-9317(02)00482-3.

[20] N. Bowden, W.T.S. Huck, K.E. Paul, G.M. Whitesides, The controlled formation of ordered, sinusoidal structures by plasma oxidation of an elastomeric 
polymer, Appl. Phys. Lett. 75 (1999) 2557-2559. doi:doi:10.1063/1.125076.

[21] S. Béfahy, P. Lipnik, T. Pardoen, C. Nascimento, B. Patris, P. Bertrand, et al., Thickness and Elastic Modulus of Plasma Treated PDMS Silica-like Surface Layer, Langmuir. 26 (2010) 3372-3375. doi:10.1021/la903154y.

[22] G. Bartalena, Y. Loosli, T. Zambelli, J.G. Snedeker, Biomaterial surface modifications can dominate cellsubstrate mechanics: the impact of PDMS plasma treatment on a quantitative assay of cell stiffness, Soft Matter. 8 (2011) 673-681. doi:10.1039/C1SM06250F.

[23] C.M. Stafford, C. Harrison, K.L. Beers, A. Karim, E.J. Amis, M.R. VanLandingham, et al., A buckling-based metrology for measuring the elastic moduli of polymeric thin films, Nat. Mater. 3 (2004) 545-550. doi:10.1038/nmat1175.

[24] S. Wang, J. Song, D.-H. Kim, Y. Huang, J.A. Rogers, Local versus global buckling of thin films on elastomeric substrates, Appl. Phys. Lett. 93 (2008) 023126-023126-3. doi:doi:10.1063/1.2956402.

[25] J. Genzer, J. Groenewold, Soft matter with hard skin: From skin wrinkles to templating and material characterization, Soft Matter. 2 (2006) 310-323. doi:10.1039/B516741H.

[26] M.-W. Moon, A. Vaziri, Surface modification of polymers using a multi-step plasma treatment, Scr. Mater. 60 (2009) 44-47. doi:10.1016/j.scriptamat.2008.08.038.

[27] Q. Cheng, Z. Sun, G. Meininger, M. Almasri, PDMS elastic micropost arrays for studying vascular smooth muscle cells, Sens. Actuators B Chem. 188 (2013) 1055-1063. doi:10.1016/j.snb.2013.08.018.

[28] S.P. Lacour, J. Jones, S. Wagner, T. Li, Z. Suo, Stretchable Interconnects for Elastic Electronic Surfaces, Proc. IEEE. 93 (2005) 1459-1467. doi:10.1109/JPROC.2005.851502.

[29] G.R. Cowper, The Shear Coefficient in Timoshenko's Beam Theory, J. Appl. Mech. 33 (1966) 335-340. doi:10.1115/1.3625046.

[30] B.E. Deal, A.S. Grove, General Relationship for the Thermal Oxidation of Silicon, J. Appl. Phys. 36 (2004) 3770-3778. doi:10.1063/1.1713945.

[31] A. Mata, A.J. Fleischman, S. Roy, Characterization of Polydimethylsiloxane (PDMS) Properties for Biomedical Micro/Nanosystems, Biomed. Microdevices. 7 (2005) 281-293. doi:10.1007/s10544005-6070-2.

[32] B. Fayolle, X. Colin, L. Audouin, J. Verdu, Relating Kinetic Models to Embrittlement in Polymer Oxidative Aging, in: Polym. Degrad. Perform., American Chemical Society, 2009: pp. 135-146. http://dx.doi.org/10.1021/bk-2009-1004.ch012 (accessed January 3, 2015).

[33] N.S. Tomer, Oxidation, Chain Scission and CrossLinking Studies of Polysiloxanes upon Ageings, Open J. Org. Polym. Mater. 02 (2012) 13-22. doi:10.4236/ojopm.2012.22003.
[34] H. Ye, Z. Gu, D.H. Gracias, Kinetics of Ultraviolet and Plasma Surface Modification of Poly(dimethylsiloxane) Probed by Sum Frequency Vibrational Spectroscopy, Langmuir. 22 (2006) 1863-1868. doi:10.1021/la052030r.

[35] S. Mani, T.M. Saif, Mechanism of controlled crack formation in thin-film dielectrics, Appl. Phys. Lett. 86 (2005) 201903. doi:10.1063/1.1927267.

[36] P.W. Alford, A.W. Feinberg, S.P. Sheehy, K.K. Parker, Biohybrid thin films for measuring contractility in engineered cardiovascular muscle, Biomaterials. 31 (2010) 3613-3621. doi:10.1016/j.biomaterials.2010.01.079.

[37] A. Grosberg, P.W. Alford, M.L. McCain, K.K. Parker, Ensembles of engineered cardiac tissues for physiological and pharmacological study: Heart on a chip, Lab. Chip. 11 (2011) 4165-4173. doi:10.1039/C1LC20557A.

[38] M.L. McCain, K.K. Parker, Mechanotransduction: the role of mechanical stress, myocyte shape, and cytoskeletal architecture on cardiac function, Pflüg. Arch. - Eur. J. Physiol. 462 (2011) 89-104. doi:10.1007/s00424-011-0951-4.

[39] A. Rabodzey, P. Alcaide, F.W. Luscinskas, B. Ladoux, Mechanical Forces Induced by the Transendothelial Migration of Human Neutrophils, Biophys. J. 95 (2008) 1428-1438. doi:10.1529/biophysj.107.119156.

[40] J.H.-C. Wang, B.P. Thampatty, An Introductory Review of Cell Mechanobiology, Biomech. Model. Mechanobiol. 5 (2006) 1-16. doi:10.1007/s10237-0050012-z. 